\title{
Do youths with a chronic condition have a different online sexual activity?
}

\author{
Marco Iuvara, MA \\ Yara Barrense-Dias, $\mathrm{PhD}$ \\ Christina Akre, PhD \\ Joan-Carles Surís, MD, MPH, PhD \\ Department of Epidemiology and Healthcare Services (DESS) \\ Center for Primary Care and Public Health (Unisanté) \\ University of Lausanne, Switzerland \\ Corresponding author: Prof. JC Suris \\ GRSA/DESS/Unisanté \\ Route de la Corniche 10 \\ 1010 Lausanne / Switzerland \\ Phone: +41213147375 \\ Fax: +41213147373 \\ Email: joan-carles.suris@unisante.ch
}

Source of funding: The survey on sexual health and behaviors of young people living in Switzerland was financed by the Swiss National Science Foundation (grant \#162538).

Conflict of Interest: The authors have no potential conflicts of interest to disclose. 
Do youths with a chronic condition have different online sexual activity?

\section{$\underline{\text { Abstract }}$}

Objective. To determine whether youths with chronic conditions differ from healthy peers in their online sexual behavior.

Methods. Youths with limiting or non-limiting chronic conditions were compared to healthy controls about their online sexual practices separately by gender.

Results. Only females with limiting conditions had significantly greater online sexual activity than healthy ones, especially for activities involving or aiming to involve a partner. Females with non-limiting conditions and all chronically ill males reported behaviors similar to those of healthy youths.

Conclusion. The anonymity offered by Internet may allow females with a limiting condition to be more confident to meet new people online.

Keywords: Chronic Disease; Online Sexual Activity; Youth; Pornography; Online Dating 
Sexual and reproductive health is important for young people's mental health. The development of the Internet and its increasing accessibility has brought a new dimension to sexuality. Moreover, its anonymity and the easy access to pornography and dating sites make it attractive for youths (Mihajlov \& Vejmelka, 2017). Online sexual activity is defined as any type of activity on the Internet that involves sexuality, from seeking information to sexual stimulation or finding a sexual partner (Giordano \& Cashwell, 2017).

Online sexual activity is on the rise. A study carried out in Germany (Beutel et al., 2017) found that $15 \%$ of respondents declared occasional online-sex use and $4 \%$ intensive use. Among 14-24 year-olds, the rates increased to 19 and 22\%, respectively. A Norwegian research among 15-20 year-olds (Gravningen, Aicken, Schirmer, \& Mercer, 2016) reported that half of girls and three out of four boys had ever used the Internet for love and sexuality. Nevertheless, it is worth noting that, among youths, not all types of online sexual behaviors are associated with the same offline sexual behaviors (Barrada, RuizGomez, Correa, \& Castro, 2019). In the Norwegian study, for example, while for most boys the main reason to find a partner on the Internet was to have sex, for girls it was mainly to start a romantic relationship (Gravningen et al., 2016). A national online survey carried out in Germany (Doring \& Mohseni, 2018) reported that being male, ayounger age and being part of a sexual minority were related to online sexual activities. In this study, $84 \%$ those aged $18-19$ years declared ever having been involved in this type of sexual activities.

Overall, online sex is more frequent among males and younger people (Beutel et al., 2017) and among those reporting a more risky sexual behavior (Gravningen et al., 2016). However, a study carried out in Israel found that, the most important factor associated with the use of online dating sites was social anxiety, and not sensationseeking or gender (Zlot, Goldstein, Cohen, \& Weinstein, 2018). 
There are some studies on Internet use among youths with a chronic condition (CC) (Lathouwers, de Moor, \& Didden, 2009; J. C. Suris, Akré, Berchtold, Bélanger, \& Michaud, 2010), but we found none on their online sexual activity. Online sexual activity may be important for CC adolescents because it allows meeting people or satisfying sexual needs with a certain degree of anonymity. Moreover, it could be hypothesized that youths with limiting CC are more prone to such activities because it may diminish their social anxiety. Additionally, it could also be hypothesized that for those with conditions limiting their daily activities that may have fewer opportunities for in-person sexual engagement, online sex may represent a good alternative to meet partners.

The objective of this paper is to compare online sexual activities of youths with a nonlimiting CC, youths with a limiting CC and youths without CC. As chronically ill youths report similar or greater sexual activity than their peers (J.-C. Suris \& Parera, 2005; Surís, Michaud, Akre, \& Sawyer, 2008), we hypothesized that they would also have similar or greater online sexual activity.

\section{Methods}

The initial sample was provided by the Swiss Federal Office of Statistics, and it was representative of the entire population living in Switzerland in terms of sex, language, and canton of residence. This sample included 49,798 individuals aged between 24 and 26 years old on 30 September 2016 (birthdate between 01 October 1989 and 30 September 1992). This age range was selected in order to be sure that the majority of the contacted people would be already sexually active and at the same time sufficiently young to be able to recall accurately the beginning of their sexual life. Among them, $2,402(4.8 \%)$ letters were returned by the postal service, because participants were not living at the address anymore, $12(0.02 \%)$ e-mails were sent by parents or caregivers to inform that the person was disabled, had gone abroad or did not speak the language, and $16(0.03 \%)$ letters were returned by participants themselves indicating that they did not want to participate. 
The final sample included 7,142 people aged between 24 and 26 years and living in Switzerland at the time when their addresses were obtained (30 September 2016). This corresponds to a response rate of $15.1 \%$. Among them 5,618 individuals completed the entire questionnaire or at least a significant part of it (78.7\% of all respondents). The mean age was 26 years and approximately half were females (49\%).(Barrense-Dias et al., 2018) Chronic conditions were defined by the answers to two questions: "Do you have a chronic illness (that is, a disease that has lasted for more than a year and that requires regular care such as asthma, diabetes, scoliosis, etc.)?" and "Do you have a physical handicap (that is, a physical problem that affects the functioning of your body, preventing you from doing the same activities as other people your age)?" with three possible answers: No; Yes, but it does not limit my daily activities; Yes, and it limits my daily activities. Those answering positively to any of the two questions were requested to indicate which condition they had and were coded according to the International Classification of Diseases (ICD) 11 th revision. Data included 980 (17\%) youths with a self-reported chronic condition. Among females, 15\% reported having a non-limiting condition and $5 \%$ a limiting one. For males, those numbers were $13 \%$ and $3 \%$, respectively. All but 37 (3.8\%) indicated their condition, with those related to the respiratory system (22\%) and to diseases of the musculoskeletal system or connective tissue $(17.8 \%)$ being the most frequent (Table 1$)$.

Additionally, all participants were asked whether they had ever "Used a dating site", "Had a rendezvous with a stranger met on the Internet", "Had sex with someone met on the Internet", "Had an erotic conversation with a stranger on the Internet" and "Had visited a pornographic website".

First, we ran a bivariate analysis comparing the three groups, using Chi2 tests for categorical variables and ANOVA for continuous ones. Then, post-hoc tests with a Bonferroni-type adjustment were made for significant online behaviors. Subsequently, we ran a multinomial regression separately for each online behavior controlling for age, 
socioeconomic level (below average/other), education level (university level/other), self-perceived health, and emotional wellbeing. Self-perceived health included five possible answers dichotomized into Good [Excellent / Very good / Good] and Poor [Fair / Poor]. We used the WHO-5 index to assess emotional wellbeing (Topp, Ostergaard, Sondergaard, \& Bech, 2015). This index includes five items referred to the last two weeks scored from 0 (at no time) to 5 (all of the time) for a total score ranging from 0 to 25. A score under 13/25 was considered as poor emotional wellbeing. We also included two sexual behavior-related variables (Ever having had a sexual partner and Ever having had sexual intercourse) to assess whether offline sexual behavior was associated with online sexual activities.

Data are presented as relative risk ratios (RRR). Analyses were performed separately by gender because differences have been observed in the sexual (J.-C. Suris \& Parera, 2005) and online (J. C. Suris et al., 2010) behaviors of males and females with CC and in online sexual behaviors such as pornography use among young people (Paul \& Shim, 2008). Nevertheless, we also performed a multinomial regression including the whole sample to assess the interaction effects as a function of gender. We used STATA14 for all analyses (Stata-Corp, College Station, Texas).

The protocol of this study was approved by the ethics committee for human research of the canton of Vaud. A complete description of the study can be found elsewhere (Barrense-Dias et al., 2018).

\section{Results}

Overall, at the bivariate level, youths with a CC tended to have a greater online sexual activity that increased with the limiting effect of the condition, although differences only reached significance among females. Post-hoc analyses showed that the differences among the female groups were mainly between those with limiting chronic conditions and the other two groups (Table 2). 
At the multivariate regression (Table 3), the only two significant differences were among females with a limiting condition for "Rendezvous with a stranger met on the Internet" (RRR=1.55) and "erotic conversation with a stranger on the Internet" $(\mathrm{RRR}=1.57)$. There was also a trend for "sex with a stranger met on the Internet" $(\mathrm{P}=0.073)$ and for "dating site" $(\mathrm{P}=0.096)$. No differences were found for males. We repeated the analyses using the whole sample and looking for interaction effects as a function of sex, but none of the interactions was significant (data not shown).

\section{Discussion}

There were no differences in online sexual behavior between healthy males and those with a CC, as seems to be the case for offline sexual behavior (J.-C. Suris \& Parera, 2005). Thereby, as males with a CC report similar sexual behavior as their healthy counterparts, they should all receive the same kind of prevention messages and counseling by health professionals. This counseling should include online activities. Among females, sexual behavior of those with a non-limiting condition was also similar to that of their healthy peers. On the contrary, females with a limiting condition had greater online sexual activity: they reported a higher rate of erotic conversations and rendezvous with strangers met on the Internet and a higher tendency to use a dating site and to have sex with someone met on the Internet. It is interesting to highlight that, unlike pornography, these four sexual activities involve or aim to involve a partner. Research shows that, online, females are looking for a relationship or a romance rather than solitary activity (Ferree, 2003). The Internet could provide a protective barrier that helps increase confidence and bypasses the dimension of physical attractiveness. Moreover, females can create a profile omitting some details they want to hide, allowing them to show an image of themselves that avoids the barrier of attractiveness (Ferree, 2003).

Females with a limiting condition seem even more attracted by these online relationships. This could be explained by the fact that, in our society, sexuality is still 
seen from a male perspective, and females could experience shame to express theirs (Ferree, 2003). Additionally, shame may be enhanced by the stigmatizing vision society can have of chronically ill people (Giordano \& Cashwell, 2017). The anonymity offered by the Internet can obviate this phenomenon and thus, make them feel more attractive. They could find themselves more desirable, and thus, be more confident communicating with and meeting new people. Furthermore, females who are often ashamed of their condition may speak more freely on the Internet, as, in general, people confide quicker online than they would do offline (Ferree, 2003). Finally, females with a CC adopt a more experimental behavioral pattern than their healthy peers (Miauton, Narring, \& Michaud, 2003), which may also explain this difference.

A previous Swiss (J. C. Suris et al., 2010) study also found that female adolescents suffering from a chronic condition, but not males, were more likely to be Internet users than their healthy peers. They hypothesized that the fact that young females used the Internet more to socialize than their male counterparts could explain their results. To some extent, our current results mirror those results in the sense that we also find differences with females with limiting CC but not among males. These gender differences need to be further studied.

This first study on online sexual activity among youths with a CC indicates a need for further research on this subject, as differences seem to exist between females with a limiting condition and their healthy peers. Future research should address whether the Internet and its anonymity are an important reason for these youth to use it. The strength of this study is that it is based on a large sample, which is representative of this population. However, some limitations need to be mentioned. First, chronic conditions and their limiting effect were self-reported, and the impact of the condition on daily activities can be subjective. Secondly, we grouped each limiting or non-limiting condition together without differentiating whether it had an impact on the youth's 
sexual development or on their Internet use (blindness or deafness, for example). Third, we do not know the frequency of these online activities in our sample.

The hypothesis that youths with a CC had greater online sexual activity was partially confirmed among females with limiting conditions. The role of the Internet as a protective barrier may play an important part in these differences. However, the other three groups had an online sexual activity similar to that of their healthy peers. These results should help clinicians and carers better target their preventive interventions and counseling regarding sexual behavior with youths with CCs, especially females with limiting ones. 


\section{References}

Barrada, J. R., Ruiz-Gomez, P., Correa, A. B., \& Castro, A. (2019). Not all Online Sexual Activities Are the Same. Front Psychol, 10, 339. doi:10.3389/fpsyg.2019.00339

Barrense-Dias, Y., Akré, C., Berchtold, A., Leeners, B., Morselli, D., \& Surís, J.-C. (2018). Sexual health and behavior of young people in Switzerland. Institut universitaire de médecine sociale et préventive (IUMSP). doi:10.16908/issn.1660-7104/291

Beutel, M. E., Giralt, S., Wolfling, K., Stobel-Richter, Y., Subic-Wrana, C., Reiner, I., . . . Brahler, E. (2017). Prevalence and determinants of online-sex use in the German population. PLoS One, 12(6), e0176449. doi:10.1371/journal.pone.0176449

Doring, N., \& Mohseni, M. R. (2018). Are Online Sexual Activities and Sexting Good for Adults' Sexual Well-Being? Results From a National Online Survey. International Journal of Sexual Health, 30(3), 250-263. doi:10.1080/19317611.2018.1491921

Ferree, M. C. (2003). Women and the web: cybersex activity and implications. Sexual and Relationship Therapy, 18(3), 385-393. doi:10.1080/1468199031000153973

Giordano, A. L., \& Cashwell, C. S. (2017). Cybersex addiction among college students: a prevalence study. Sexual Addiction \& Compulsivity, 24(1-2), 47-57. doi:10.1080/10720162.2017.1287612

Gravningen, K., Aicken, C. R., Schirmer, H., \& Mercer, C. H. (2016). Meeting sexual partners online: associated sexual behaviour and prevalent chlamydia infection among adolescents in Norway: a cross-sectional study. Sex Transm Infect, 92(2), 97-103. doi:10.1136/sextrans-2015-052152

Lathouwers, K., de Moor, J., \& Didden, R. (2009). Access to and use of Internet by adolescents who have a physical disability: a comparative study. Research in Developmental Disabilities, 30(4), 702-711. doi:10.1016/j.ridd.2008.09.003

Miauton, L., Narring, F., \& Michaud, P.-A. (2003). Chronic illness, life style and emotional health in adolescence: results of a cross-sectional survey on the health of 15-20year-olds in Switzerland. European Journal of Pediatrics, 162(10), 682-689. doi:10.1007/s00431-003-1179-x

Mihajlov, M., \& Vejmelka, L. (2017). Internet addiction: a review of the first twenty years. Psychiatria Danubina, 29(3), 260-272. doi:10.24869/psyd.2017.260

Paul, B., \& Shim, J. W. (2008). Gender, Sexual Affect, and Motivations for Internet Pornography Use. International Journal of Sexual Health, 20(3), 187-199. doi:10.1080/19317610802240154

Suris, J.-C., \& Parera, N. (2005). Sex, drugs and chronic illness: health behaviours among chronically ill youth. European Journal of Public Health, 15(5), 484-488. doi:10.1093/eurpub/cki001

Suris, J. C., Akré, C., Berchtold, A., Bélanger, R. E., \& Michaud, P. A. (2010). Chronically connected? Internet use among adolescents with chronic conditions. Journal of Adolescent Health, 46(2), 200-202. doi:10.1016/j.jadohealth.2009.07.008

Surís, J. C., Michaud, P., Akre, C., \& Sawyer, S. M. (2008). Health risk behaviors in adolescents with chronic conditions. Pediatrics, 122(5), e1113-e1118. doi:10.1542/peds.2008-1479

Topp, C. W., Ostergaard, S. D., Sondergaard, S., \& Bech, P. (2015). The WHO-5 Well-Being Index: a systematic review of the literature. Psychother Psychosom, 84(3), 167176. doi:10.1159/000376585

Zlot, Y., Goldstein, M., Cohen, K., \& Weinstein, A. (2018). Online dating is associated with sex addiction and social anxiety. J Behav Addict, 7(3), 821-826.

doi:10.1556/2006.7.2018.66 